\title{
TRP1(0RF3):1-9 Peptide
}

National Cancer Institute

\section{Source}

National Cancer Institute. TRP1(ORF3):1-9 Peptide. NCI Thesaurus. Code C2816.

A recombinant peptide consisting of amino acid residues 1 to 9 of the tyrosinase-related protein 1 (TRP1). Expressed by cells of melanocyte origin, TRP1 is an enzyme involved in the process that converts tyrosinase to melanin pigments. Vaccination with TRP1(ORF3):1-9 may stimulate cytotoxic T cell responses to melanoma cells.(NCI04) 